\title{
Determinación del potencial de membrana mitocondrial mediante citometría de flujo durante el proceso de criopreservación de espermatozoides epididimarios de alpacas
}

\section{Determination of mitochondrial membrane potential by flow cytometry during the cryopreservation process of epididymal alpaca spermatozoa}

\author{
Pablo Allauca ${ }^{1}$, Alejandra Ugarelli ${ }^{1}$, Alexei Santiani ${ }^{1}$
}

\section{Resumen}

\begin{abstract}
El objetivo de este estudio fue demostrar, mediante la citometría de flujo, que el porcentaje de espermatozoides de alpaca con alto potencial de membrana mitocondrial (PMM) se reduce significativamente luego del proceso de criopreservación. Se trabajó con 41 testículos de alpacas obtenidos del Camal Municipal de Ninacaca, Pasco, Perú. Se recuperaron los espermatozoides de la cola de los epidídimos con un dilutor en base a leche descremada y se congelaron solo aquellas muestras con mínimos de $30 \%$ de motilidad y $50 \times 10^{6}$ espermatozoides $/ \mathrm{ml}$. La evaluación del PMM se realizó antes y después del proceso de criopreservación. Cada muestra se incubó durante 10 minutos a $38^{\circ} \mathrm{C}$ con MitoTracker Deep Red (100 nM) para determinar el porcentaje de alto PMM, mediante citometría de flujo con imágenes. El efecto de la criopreservación en el porcentaje de espermatozoides con alto PMM fue evaluado mediante la prueba de T-student pareada. Así mismo, se correlacionaron los porcentajes de motilidad y espermatozoides con alto PMM. Se obtuvo un $49.82 \pm 12.41$ y de $34.97 \pm 9.96 \%$ de alto PMM en muestras antes y después del proceso de criopreservación $(\mathrm{p}<0.05)$. Se encontró una correlación positiva $(\mathrm{r}=0.62 ; \mathrm{p}<0.0001)$ entre motilidad y PMM. Se concluye que el PMM se reduce significativamente luego del proceso de criopreservación, parámetro relacionado a la motilidad espermática.
\end{abstract}

Palabras clave: alpaca; espermatozoide; potencial de membrana mitocondrial; MitoTracker Deep Red 633

${ }^{1}$ Laboratorio de Reproducción Animal, Facultad de Medicina Veterinaria, Universidad Nacional Mayor de San Marcos, Lima, Perú

${ }^{2}$ E-mail: asantiania@unmsm.edu.pe

Recibido: 27 de abril de 2018

Aceptado para publicación: 18 de octubre de 2018 
The aim of this study was to demonstrate, by flow cytometry, that the percentage of alpaca spermatozoa with high mitochondrial membrane potential (MMP) is significantly reduced after the cryopreservation process. Forty-one alpaca testicles were obtained from a local slaughterhouse in the Ninacaca district, Pasco, Peru. The spermatozoa were recovered from the tail of the epididymis with an extender based on skim milk and only those samples with minimum $30 \%$ motility and $50 \times 10^{6}$ spermatozoa/ml were cryopreserved. The MMP evaluation was carried out before and after the cryopreservation process. Each sample was incubated for 10 minutes at $38^{\circ} \mathrm{C}$ with MitoTracker Deep Red (100 nM) to determine the percentage of high MMP by imaging flow cytometry. The effect of cryopreservation on the percentage of spermatozoa with high MMP was evaluated by a paired t-student test and the percentage of motility and sperm with high MMP were correlated. A $49.82 \pm 12.41$ and $34.97 \pm 9.96 \%$ of high MMP were obtained in samples before and after the cryopreservation process $(\mathrm{p}<0.05)$. A positive correlation was found $(\mathrm{r}=0.62, \mathrm{p}<0.0001)$ between motility and PMM. It is concluded that MMP is significantly reduced after the cryopreservation process, a parameter related to sperm motility.

Key words: alpaca; spermatozoa; mitochondrial membrane potential; MitoTracker Deep Red 633

\section{INTRODUCCIÓN}

El empleo de biotecnologías reproductivas viene permitiendo lograr animales genéticamente superiores, así como reducir el tiempo generacional en varias especies de interés ganadero. Sin embargo, la complejidad en las características fisiológicas reproductivas de la alpaca no ha permitido la utilidad deseada en esta especie (Fernández-Baca, 1993). Para su mejora productiva se requiere la distribución de material genético de alta calidad hacia distintas áreas geográficas que puedan beneficiarse con la obtención de crías mejoradas. Es así que se necesita evaluar diversos parámetros del material genético; entre ellos, el volumen, $\mathrm{pH}$, motilidad, concentración, morfología y viabilidad espermática. Actualmente, un nuevo parámetro a utilizar es el potencial de membrana mitocondrial (PMM) de los espermatozoides, el cual podría ser un indicador directo de motilidad e indirecto de capacidad fecundante (Manosalva et al., 2005). El interés en evaluar la actividad mitocondrial deriva de su importancia en la producción de adenosina-tri-fosfato (ATP), producido por fosforilación oxidativa y necesario para la motilidad y la fecundación (Andrade, 2005).

Recientemente, la técnica de citometría de flujo ha ofrecido una mayor precisión en la forma de medir la calidad del semen (Hallap et al., 2005), incluyendo la determinación del porcentaje de espermatozoides con alto PMM como parámetro en diversas especies, además de la alpaca (Cheuquemán et al., 2013). Una de las tinciones específicas para mitocondrias es el MitoTraker ${ }^{\circledR}$ Deep Red, cuyas moléculas se difunden a través de la membrana plasmática y se unen específicamente a los lípidos de membrana de las mitocondrias funcionales. Las principales ventajas son que presentan especificidad, fotoestabilidad, y elevada sensibilidad al ser incorporado en distintos protocolos.

Reportes de la literatura científica indican que Santiani et al. (2016) evaluaron el PMM en espermatozoides frescos epididimarios de alpaca con los fluorocromos MitoTracker ${ }^{\circledR}$ Deep Red y Mito Tracker CMXRos, así como el trabajo de Cheuqueman et al. 
(2013), quienes evaluaron el PMM en muestras de eyaculado de alpacas utilizando el fluorocromo JC-1. Adicionalmente, se tiene el reporte de Santiani et al. (2015) donde evaluaron muestras descongeladas epididimarias de alpaca, utilizando en este caso el fluorocromo MitoTracker CMXRos. Sin embargo, se desconoce el efecto de la criopreservación sobre el PMM en espermatozoides de alpaca. Se ha demostrado que la criopreservación afecta negativamente diversos parámetros del funcionamiento espermático, por lo que podría esperarse que el porcentaje de espermatozoides con alto PMM se vea afectado por este proceso (Castelo et al., 2008). Por lo tanto, el objetivo del presente estudio fue determinar, mediante la citometría de flujo, el efecto del proceso de criopreservación sobre el porcentaje de espermatozoides de alpaca con alto PMM.

\section{Materiales y MéTodos}

\section{Muestras}

Las muestras se obtuvieron del Camal Municipal del Distrito de Ninacaca, departamento de Pasco, Perú, y se procesaron en el Laboratorio de Reproducción Animal de la Facultad de Medicina Veterinaria de la Universidad Nacional Mayor de San Marcos, Lima, durante el año 2016.

El tamaño muestral se calculó utilizando la fórmula $\mathrm{n}=\left(2\left(\mathrm{Z}_{\alpha}+\mathrm{Z}_{\beta}\right)^{2 *} \mathrm{~S}^{2} / \mathrm{d}^{2}\right.$, considerando un valor de $Z_{\alpha}$ de $1.645, Z_{\beta}$ de 1.282 , $\mathrm{S}^{2}=19 \%$ y d $=15 \%$, lo cual dio como resultado una $\mathrm{n}$ de 41 testículos. Los testículos se recuperaron luego del beneficio de los animales, se lavaron con solución fisiológica $(\mathrm{NaCl}$ $0.9 \%)$ y se colocaron en bolsas herméticas individuales junto con la solución fisiológica. Se almacenaron en cajas transportadoras, utilizando un gel refrigerante para mantener la temperatura a $5{ }^{\circ} \mathrm{C}$ y se trasladaron al laboratorio. Los testículos debían tener un tamaño mínimo de $3 \mathrm{~cm}$ y un peso mínimo de $10 \mathrm{~g}$ para ser considerarlos en el presente estudio, dado que son adecuados indicadores de la función espermática de un macho adulto (Abraham et al., 2016).

\section{Dilutor}

Se mezcló $19 \mathrm{ml}$ de leche descremada, $1 \mathrm{ml}$ de yema de huevo y $0.97 \mathrm{~g}$ de fructosa. A esta mezcla se le añadió $1840 \mu 1 \mathrm{del}$ crioprotector dimetilacetamida (DMA), equivalente a una concentración final $1 \mathrm{M}$. Esta mezcla final se mantuvo temperada a $37.5^{\circ} \mathrm{C}$.

\section{Espermatozoides Epididimarios}

Se retiró la túnica vaginal visceral de cada testículo, se hizo la divulsión para separar el epidídimo y se aisló la cola del epidídimo mediante un corte con tijera Mayo recta y pinza de Adson. Se retiró todo el tejido conectivo visible con el mismo instrumental y con el bisel no cortante de una hoja de bisturí se corrió el contenido de los vasos sanguíneos para evitar la contaminación de la muestra. Antes de aislar la cola del epidídimo, se realizó un lavado con PBS y luego se colocó la cola del epidídimo en una placa Petri temperada a $37.5^{\circ} \mathrm{C}$, y se agregó $1 \mathrm{ml}$ de dilutor para proceder a la liberación, mediante cortes seriados de la cola del epidídimo. Finalmente, se colectó la suspensión en un tubo eppendorf de $1.5 \mathrm{ml}$ y se mantuvo temperado a $37.5^{\circ} \mathrm{C}$.

\section{Calidad Seminal}

La motilidad fue evaluada en forma subjetiva, utilizando $10 \mu 1$ de cada muestra de suspensión de espermatozoides recuperados. Se colocó la muestra sobre una lámina porta objeto temperada a $37.5^{\circ} \mathrm{C}$, se cubrió con una lámina cubre objeto y se observó en el microscopio con un objetivo 40X. Para la concentración de la suspensión de espermatozoides recuperados cada muestra se diluyó en una proporción 1:20 con agua destilada, se recuperó $10 \mu 1$ de cada dilución y se leyó en la cámara de Neubauer a 400X. Solo aquellas muestras con motilidad $\geq 30 \%$ $y$ concentración $\geq 50 \times 10^{6}$ espermatozoides por $\mathrm{ml}$ fueron procesados. 


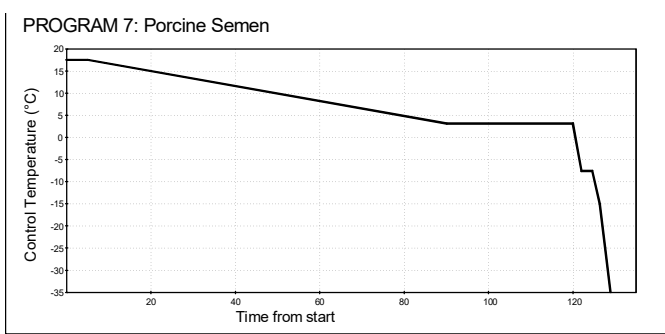

Figura 1. Programa \#7 de congelamiento automático Cryobath (Cryologic)

\section{Criopreservación}

Para el proceso de criopreservación se utilizó nitrógeno líquido dentro de un sistema de congelamiento automático (Cryobath, Cryologic). Las muestras de espermatozoides suspendidas en el dilutor se envasaron en pajillas de plástico de $0.25 \mathrm{ml}$. Se utilizó el programa \# 7 del sistema (Figura 1), el cual se inició a una temperatura de $18^{\circ} \mathrm{C}$, disminuyendo hasta llegar a $5{ }^{\circ} \mathrm{C}$ en un periodo de 90 minutos, para luego mantenerse en esas condiciones por 30 minutos. Luego, la temperatura descendió hasta lograr la congelación de las pajillas. Estas fueron luego colocadas en nitrógeno líquido hasta su evaluación.

\section{Motilidad Pos-descongelación}

Las pajillas se descongelaron en baño maría a $37{ }^{\circ} \mathrm{C}$ durante un minuto, luego se secaron y el contenido de cada pajilla se colocó en un tubo de $1.5 \mathrm{ml}$. Se tomó $10 \mu \mathrm{lde}$ cada muestra y se colocó en una lámina porta objeto temperada a $37.5^{\circ} \mathrm{C}$, se cubrió con una lámina cubre objeto y se observó en el microscopio con un objetivo $40 \mathrm{X}$ para evaluar la motilidad.

\section{Potencial de Membrana Mitocondrial (PMM)}

El PPM se evaluó utilizando el fluorocromo MitoTracker Deep Red (M22425, Molecular Probes, EEUU). Se rea- lizó antes y después del proceso de criopreservación. Cada muestra fresca y descongelada se lavó dos veces por centrifugación con PBS a $600 \mathrm{~g}$ por $8 \mathrm{~min}$. Se eliminó el sobrenadante y el pellet se resuspendió en $150 \mu 1$ de PBS. Se preparó una solución stock de MitoTracker Deep Red, para lo cual se diluyó $50 \mu \mathrm{g}$ en $92 \mu \mathrm{l}$ de DMSO para llegar a una concentración de $1 \mathrm{mM}$. Se procedió a homogenizar y se prepararon alícuotas de $2 \mu 1$ guardándolas en congelación. Luego se preparó una solución de trabajo para lo cual se diluyó $2 \mu 1$ de solución stock de MitoTracker Deep Red con $100 \mu 1$ de PBS, para llegar a una concentración de $20 \mu \mathrm{M}$. Se tomó $100 \mu \mathrm{l}$ de muestra después del lavado y se agregó $0.5 \mu 1$ de solución de trabajo de MitoTracker Deep Red 633 $(20 \mu \mathrm{M})$ para obtener una concentración final de $100 \mathrm{nM}$. Se incubó durante $10 \mathrm{~min}$ a $38{ }^{\circ} \mathrm{C}$ en oscuridad.

\section{Citometría de Flujo}

Las muestras se analizaron utilizando un citómetro de flujo FlowSight (Amnis) equipado con un sistema analizador de imágenes. Se leyeron diez mil espermatozoides por cada muestra. Para la excitación del MitoTracker Deep Red se utilizó un láser de longitud de onda de $642 \mathrm{~nm}$ y la emisión de la fluorescencia se leyó utilizando el canal de detección 11 (Ch-11): (642-740 nm). Los eventos se presentaron utilizando un gráfico tipo histograma, en donde el eje «X» midió la intensidad de fluorescencia del Ch-11 y en el eje «Y» la frecuencia de los eventos. Se consideraron espermatozoides con actividad mitocondrial a aquellos que presentaron fluorescencia roja. Los resultados se expresaron en porcentaje de espermatozoides con actividad mitocondrial.

Dado que el citómetro de flujo cuenta con un sistema análogo de imagen, se observaron fotos de espermatozoides con alto y bajo PMM de los siguientes canales:

Ch1: Presentado en campo claro (longitud de onda: 435-505 nM) 


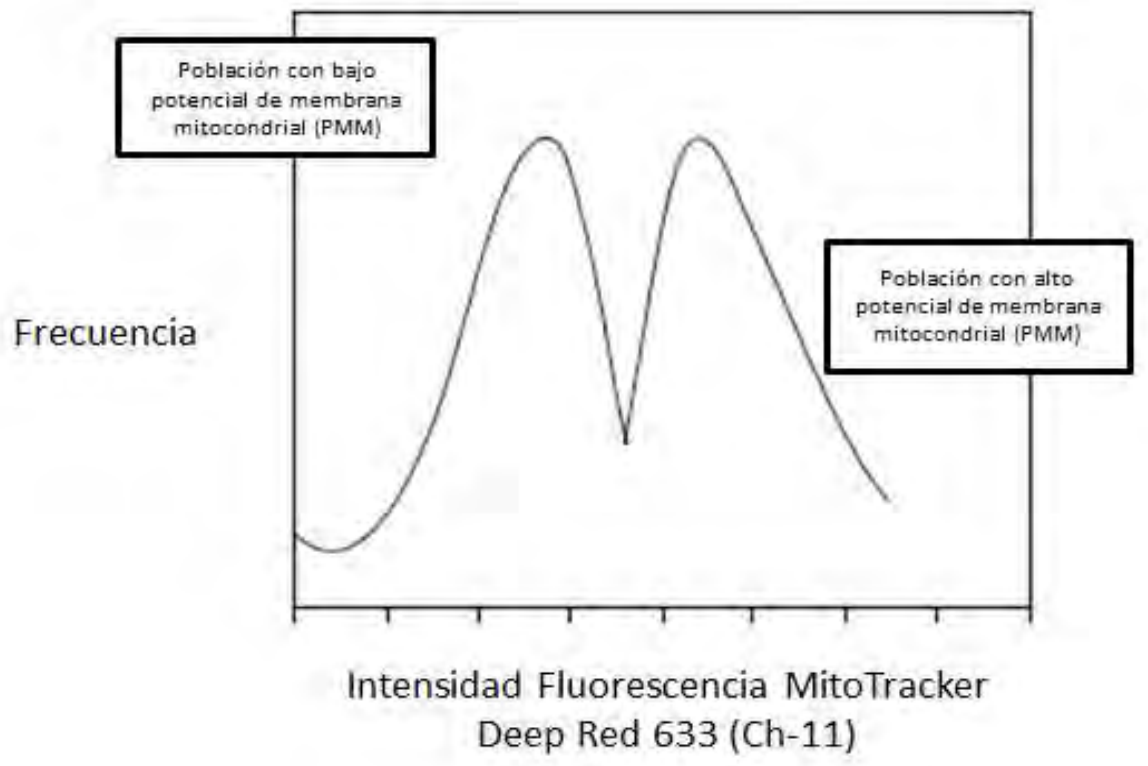

Figura 2. Esquema de presentación de histograma de espermatozoides. Muestra dos poblaciones diferenciadas de espermatozoides

Ch11: Presentado en campo oscuro (longitud de onda: $642-745 \mathrm{nM}$ )

Ch1/Ch11: Presentado en campo claro y oscuro (longitud de onda: $435-505 \mathrm{nM}+$ $642-745 \mathrm{nM})$

\section{Controles de Fluorescencia}

Para la corroboración de los datos se utilizaron controles de autofluorescencia, control negativo y control positivo. Los datos se presentaron en tres histogramas: el control de autofluorescencia en donde se analizó las muestras espermáticas a través del citómetro de flujo sin la utilización de algún fluorocromo; el control negativo, donde las muestras de espermatozoides se indujeron a muerte celular, y luego se evaluaron a través del citómetro de flujo utilizando el fluorocromo MitoTracker Deep Red; y el control positivo, donde una muestra de espermatozoides a condiciones normales fue evaluada a través del citómetro de flujo utilizando el fluorocromo MitoTracker Deep Red 633.

\section{Diseño Experimentañ y Análisis}

Se evaluaron las 41 muestras obtenidas, divididas en dos etapas: evaluación de muestras en fresco y evaluación post congelamiento. El promedio de los porcentajes de espermatozoides con alto PMM, frescos y descongelados, se analizaron mediante un tStudent de muestras pareadas. Además, los datos se presentaron como media, mediana, coeficiente de variación e intervalo de confianza. Los porcentajes de motilidad y PMM también fueron comparados utilizando la Correlación de Pearson. Se utilizó el programa GraphPad Prism ${ }^{\circledR}$ v. 3.0.

\section{Resultados}

Los datos estadísticos de los espermatozoides que presentan alto potencial de membrana mitocondrial (PMM) se presentan en el Cuadro 1. El $49.82 \pm 12.41 \%$ de espermatozoides frescos y el $34.97 \pm 9.96 \%$ de esper- 
Cuadro 1. Espermatozoides epididimarios de alpacas, frescos y descongelados, analizados con el fluorocromo MitoTracker Deep Red, con alto potencial de membrana mitocondrial (PMM)

\begin{tabular}{lcc}
\hline & $\begin{array}{c}\text { Muestras } \\
\text { frescas } \\
(\%)\end{array}$ & $\begin{array}{c}\text { Muestras } \\
\text { descongeladas } \\
(\%)\end{array}$ \\
\hline Promedio & 49.82 & 34.97 \\
Mediana & 51.50 & 35.90 \\
$\begin{array}{l}\text { Desviación } \\
\text { estándar }\end{array}$ & 12.41 & 9.95 \\
$\begin{array}{l}\text { Coeficiente } \\
\text { de variación }\end{array}$ & 24.91 & 28.48 \\
$\begin{array}{l}\text { Intervalo de } \\
\text { confianza }\end{array}$ & 7.84 & 6.29 \\
\hline
\end{tabular}

matozoides descongelados presenta alto PMM, siendo esta diferencia estadísticamente significativa $(\mathrm{p}<0.05)$ mediante la prueba de $t$ de Student (Cuadro 2). En forma similar, la motilidad fue de $46.10 \pm 7.71 \%$ en las muestras de espermatozoides frescos y de $24.07 \pm 6.50 \%$ en las muestras de espermatozoides descongelados, habiendo diferencias significativas $(\mathrm{p}<0.05)$ entre grupos (Cuadro 2).

Cuadro 2. Potencial de membrana mitocondrial (PMM) y la motilidad de espermatozoides epididimarios de alpaca, frescos y descongelados

\begin{tabular}{ccc}
\hline & Frescos & Descongelados \\
\hline $\begin{array}{c}\text { Alto PMM } \\
(\%)\end{array}$ & $49.82 \pm 12.41^{\mathrm{a}}$ & $34.97 \pm 9.96^{\mathrm{b}}$ \\
$\begin{array}{c}\text { Motilidad } \\
(\%)\end{array}$ & $46.10 \pm 7.71^{\mathrm{a}}$ & $24.07 \pm 6.50^{\mathrm{b}}$ \\
\hline $\begin{array}{l}\text { a,b } \\
\text { Letras diferentes dentro de líneas indican }\end{array}$ \\
diferencia estadística significativa $(p<0.05)$
\end{tabular}

En la Figura 3 se presentan ejemplos gráficos (histogramas) del análisis de las muestras de espermatozoides epidimarios de alpaca mediante el citómetro de flujo. En (A) se presenta el control de autofluorescencia del fluorocromo MitoTracker Deep Red, donde se muestra que no hay emisión de fluoresencia en Ch 11, por lo que todos los eventos forman una sola población ubicada a la izquierda del eje $\mathrm{x}$, indicando bajo PMM. En (B) se presenta el control negativo, espermatozoides inducidos a muerte celular analizados utilizando el fluorocromo MitoTracker Deep Red, donde se observa que los eventos forman una sola población ubicada a la izquierda del eje $\mathrm{x}$, indicando bajo potencial de membrana mitocondrial. En (C) y (D) se presentan el análisis de muestras frescas y muestras descongeladas, respectivamente. En (C) se puede observar una mayor distribución de eventos localizados a la derecha del eje x y en (D) se puede observar una mayor distribución de eventos localizados a la izquierda del eje $\mathrm{x}$, por lo cual representan una variación en las poblaciones antes y después del proceso de criopreservación.

En la Figura 4 se presentan fotos de espermatozoides analizados mediante citometría de flujo. El espermatozoide que se observa en la Figura 4A pertenece a la población de eventos con alto potencial de membrana mitocondrial, observada mediante el sistema analizador de imágenes del citómetro de flujo utilizando el canal Ch 1 en campo claro. Se presenta con fluorecencia naranja intensa ante la exposición a los filtros para el fluorocromo MitoTracker Deep Red del Ch 11 en campo oscuro y utilizando los canales Ch 1 y Ch 11 en campo claro y oscuro. En la Figura 4B se presenta un espermatozoide que pertenece a la población de eventos con bajo potencial de membrana mitocondrial, observada bajo el sistema analizador de imágenes del citómetro de flujo utilizando el Ch 1 en campo claro, y observado ante la exposición a los filtros para el fluorocromo MitoTracker Deep Red del canal Ch 11 en campo oscuro y utilizando los canales Ch 1 y Ch 11 en campo claro y oscuro. 
A)

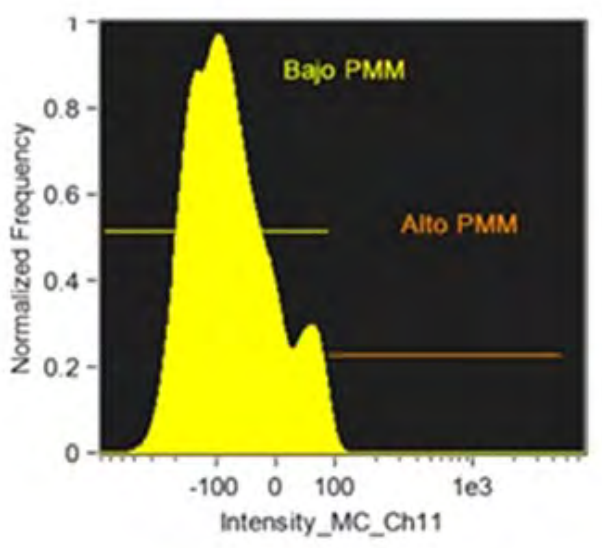

C)

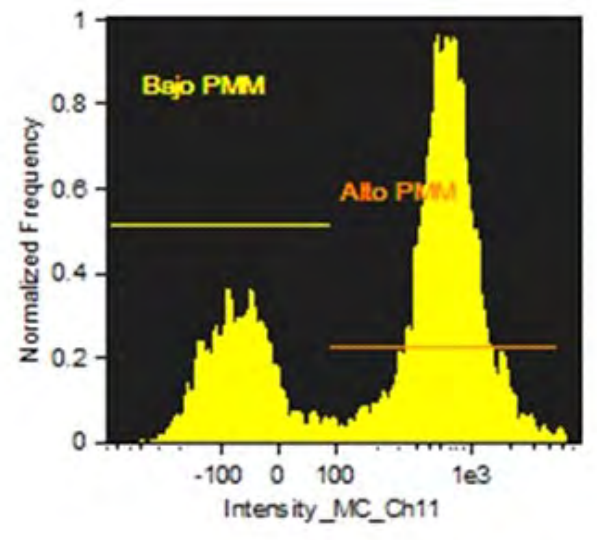

B)

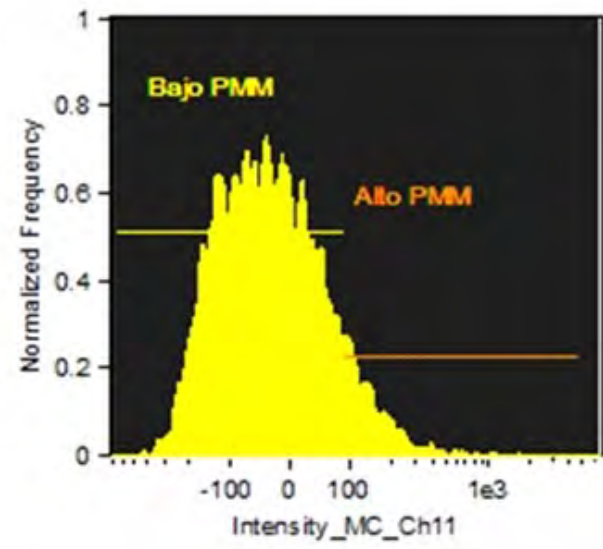

D)

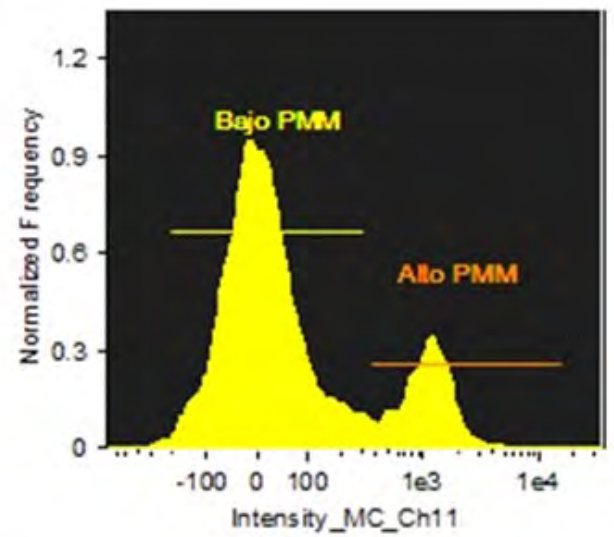

Figura 3. Histogramas de muestras de espermatozoides epididimarios de alpaca analizados mediante el citómetro de flujo. (A) Control de autofluorescencia, muestra de espermatozoides analizados sin utilizar el fluorocromo MitoTracker Deep Red. (B) Control negativo, se analizó espermatozoides inducidos a muerte celular, utilizando el fluorocromo MitoTracker Deep Red. (C) y (D) representan muestras de espermatozoides epididimarios frescos y descongelados, respectivamente, utilizando el fluorocromo MitoTracker Deep Red

El análisis de correlación de Pearson entre los valores porcentuales obtenidos entre motilidad y el porcentaje de alto PMM $(\mathrm{n}=82$ pares) indicó una correlación positiva significativa $(\mathrm{p}<0.0001)$ con un valor de $\mathrm{r}=$ 0.6271 (Figura 5).

\section{Discusión}

Este trabajo es el primer reporte que confirma que el PMM disminuye significativamente durante el proceso de criopreservación en espermatozoides 
(A)

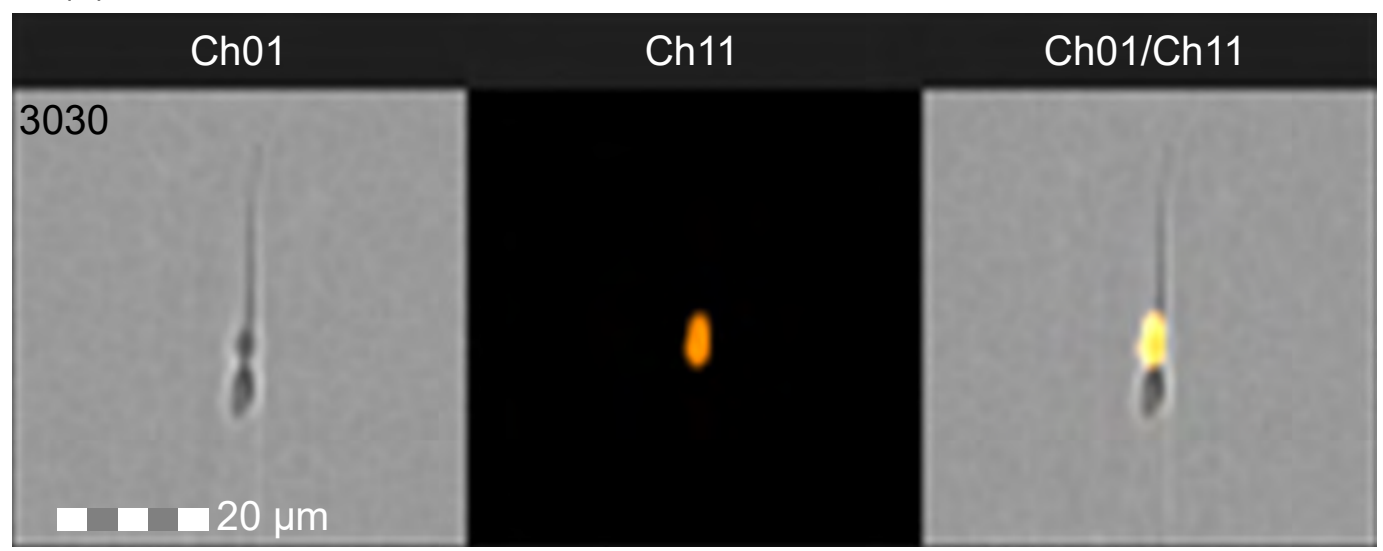

(B)

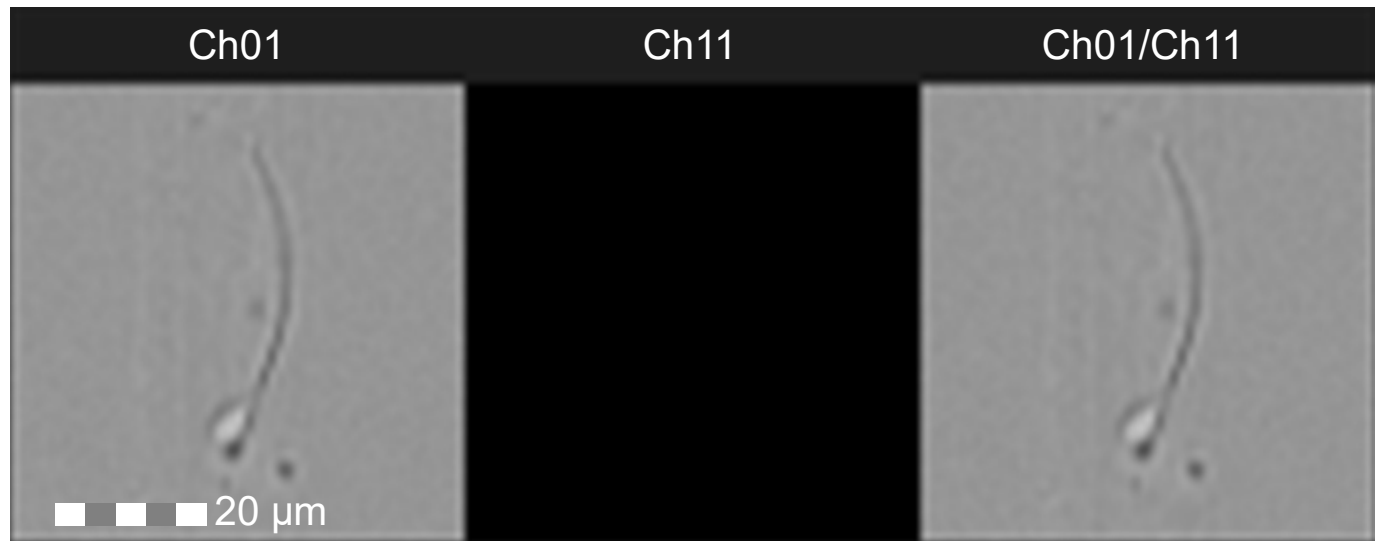

Figura 4: Ejemplos de espermatozoides epididimarios de alpaca evaluados mediante el sistema analizador de imagen del citómetro de flujo. (A) Espermatozoide de alto potencial de membrana mitocondrial (PMM). (B) Espermatozoide de bajo PMM

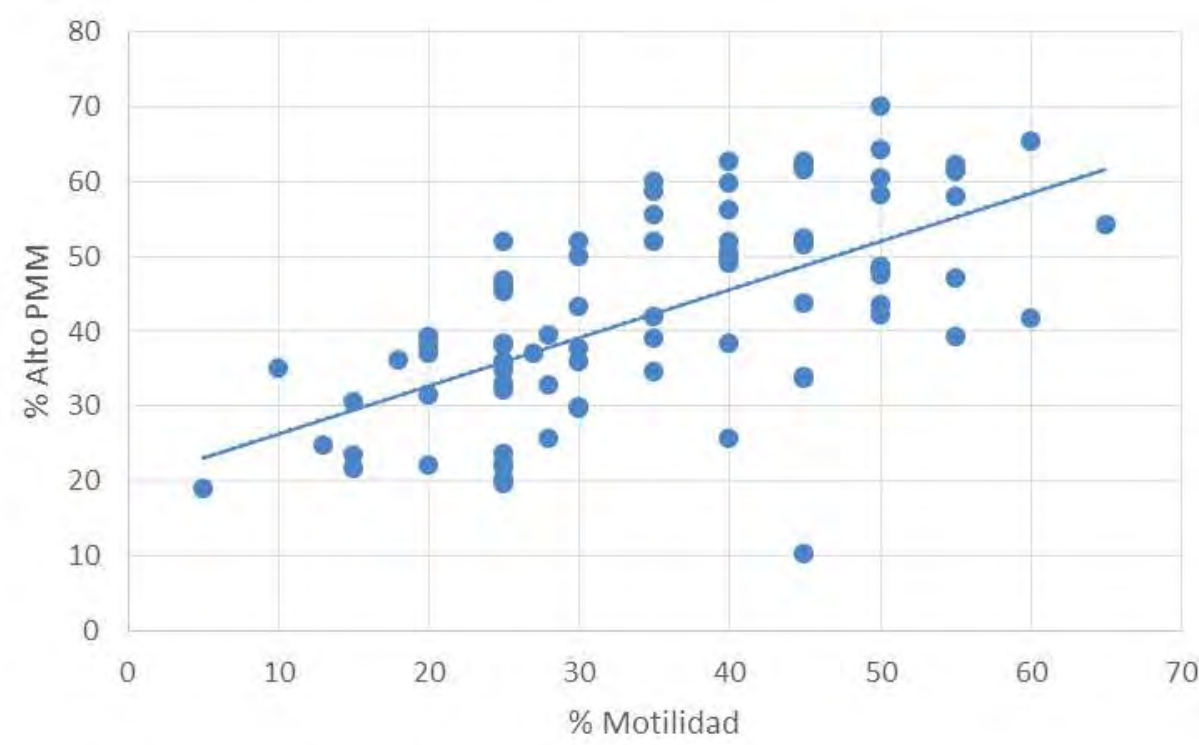

Figura 5. Correlación entre el porcentaje de motilidad y alto potencial de membrana mitocondrial (PMM) de espermatozoides epididimarios de alpaca 
epididimarios de alpacas analizados mediante el citómetro de flujo y utilizando el fluorocromo MitoTracker Deep Red 633. El PMM es la diferencia de voltaje a través de la membrana mitocondrial, el cual es un componente importante de la fuerza protón-motriz en las mitocondrias que se utiliza para realizar la síntesis de ATP (Vojtísková et al., 2004).

Se encontraron valores cercanos al 50\% de alto PMM en muestras espermáticas frescas, lo cual fue ligeramente inferior al descrito previamente por Santiani et al. (2016), donde obtuvieron alrededor de $59 \%$ de alto PMM, utilizando también el fluorocromo MitoTracker Deep Red 633. En dicho trabajo también evaluaron espermatozoides utilizando el flurocromo CMXRos obteniendo valores de $65 \%$ de alto PMM. Es posible que el factor estacional haya influido en los resultados, dado que el presente estudio se hizo en los meses de verano (enero-mayo), mientras que Santiani et al. (2016) trabajaron en los meses de primavera (septiembre-enero), considerando que Urquieta et al. (1994) menciona que los meses de mayor reproducción corresponden entre marzo y mayo. Por otro lado Cheuqueman et al. (2013) evaluaron muestras de eyaculado de alpaca utilizando el fluorocromo JC-1 obteniendo un valor de $66 \%$ de alto PMM utilizando seis alpacas de comprobada fertilidad, en tanto que en el presente estudio se utilizaron testículos de alpacas beneficiadas.

La calidad espermática disminuyó significativamente por el proceso de criopreservación, pues descendió a 35\% de alto PMM. Resultados similares obtuvieron Santiani et al. (2015), encontrando un 25\% de alto PMM en espermatozoides epididimarios de alpaca, utilizando en este caso el fluorocromo MitoTracker Red CMXRos. En otras especies, Hallap et al. (2005) utilizando también el fluorocromo MitoTracker Deep Red encontraron 69\% de alto PMM en espermatozoides frescos de toros y alrededor de $48 \%$ en espermatozoides descongelados.
Con respecto a la motilidad, las muestras de espermatozoides frescos obtuvieron un valor de $46 \%$ y las muestras de espermatozoides descongelados un valor de 24\%. En el estudio de Valdivia et al. (1999) con muestras seminales de alpaca obtuvieron también una variación considerable en la motilidad, siendo de $60-98 \%$ en muestras frescas y de $15-20 \%$ luego del proceso de congelación-descongelación. Así mismo, Santiani et al. (2005) utilizando muestras seminales recolectadas por vagina artificial obtuvieron motilidades de $72 \%$ en muestras frescas y $20 \%$ en muestras descongeladas. Cabe resaltar que en dicho estudio, se trabajó con muestras de cuatro alpacas criadas bajo buena alimentación, lo cual puede explicar los mayores valores de motilidad respecto a los del presente estudio. Por otro lado, los mejores valores de motilidad pos-descongelación entre ambos estudios se puede deber a las técnicas de congelación, ya que el método automático utilizado en el presente trabajo minimiza el daño celular durante la criopreservación. En diversos estudios se ha demostrado la pérdida de motilidad por efecto de la criopreservación (Aller et al., 2003; Piomboni et al., 2012).

Tal como indica O'Connell et al. (2003), existe una relación directa entre motilidad y PMM. La correlación de Pearson entre la motilidad y el PMM en el presente trabajo fue altamente significativa $(\mathrm{p}<0.0001)$.

\section{Conclusiones}

- El potencial de membrana mitocondrial (PMM) de las muestras espermáticas se reduce por efecto del proceso de criopreservación.

- El PMM es un parámetro de calidad espermática que tiene directa relación con la motilidad. 


\section{Agradecimientos}

Se agradece al proyecto $\mathrm{N} .{ }^{\circ} 123-$ FINCyT-ECL-2014 por el financiamiento para el estudio.

\section{Literatura Citada}

1. Abraham MC, Puhakka J, Ruete A, Al-Essawe EM, de Verdier K, Morrel JM, Gage R. 2016. Testicular length as an indicator of the onset of sperm production in alpacas under Swedish conditions. Acta Vet Scand 58: 10. doi: 10.1186/s13028-016-0191-x

2. Aller JF, Ferre L, Rebuffi G, Alberio RH. 1997. Recolección de semen de llama (Lama glama) en la Puna argentina. Vet Arg 14: 104-107.

3. Andrade A. 2005. Influencia en la calidad espermática de la adición de distintas concentraciones de crioprotectores para la conservación del semen canino. Tesis Doctoral. Madrid: Univ. Complutense de Madrid. $287 \mathrm{p}$.

4. Castelo T, Rodríguez T, Rodriguez A. 2008. Considerations on goat semen cryopreservation. Acta Vet Bras 2: 67-75.

5. Cheuquemán C, Merino O, Giojalas L, Von Baer A, Sánchez R, Risopatrón $J$. 2013. Assessment of sperm function parameters and DNA fragmentation in ejaculated alpaca sperm (Lama pacos) by flow cytometry. Reprod Domest Anim 48: 447-453. doi: 10.1111/rda.12096

6. Fernández-Baca S. 1993. Manipulation of reproductive functions in male and female new world camelids. Anim Reprod Sci 33: 307-323. doi: 10.1016/ 0378-4320(93)90121-7

7. Hallap T, Nagy S, Jaakma U, Johannisson A, Rodríguez-Martínez H. 2005. Mitochondrial activity of frozen-thawed spermatozoa assessed by Mitotracker Deep Red 633. Theriogenology 63: 2311-2322. doi: 10.1016/ j.theriogenology.2004.10.010
8. Manosalva I, Cortés C, Leyva V, Valdivia M, De los Reyes M, Barros C, Moreno R. 2005. Efecto de la refrigeración sobre la motilidad, integridad de membrana acrosomal y reacción acrosomal en espermatozoides caninos. Rev Inv Vet Peru 16: 114-128. doi: 10.15381/ rivep.v16i2.1548

9. O'Connell M, McClure N, Powell L, Steele E, Lewis S. 2003. Differences in mitochondrial and nuclear DNA status of high-density and low-density sperm fractions after density centrifugation preparation. Fertil Steril 79: 754-762. doi: 10.1016/S0015-0282(02)04827-6

10. Piomboni P, Focarelli R, Stendardi A, Ferramosca A, Zara V. 2012. The role of mitochondria in energy production for sperm motility. Int J Androl 35: 109-124. doi: 10.1111/j.1365-2605.2011.01218.x

11. Santiani A, Huanca W, Sapana $R$, Huanca T, Sepúlveda N, Sánchez R. 2005. Effects on the quality of frozenthawed alpaca (Lama pacos) semen using two different cryoprotectans and extenders. Asian J Androl 7: 303-309. doi: 10.1111/j.1745-7262.2005.00021.x

12. Santiani A, Ugarelli A, Evangelista $S$, Choez K, Pacheco J. 2015. Evaluación de diferentes concentraciones de FITCPSA y FITC-PNA para la valoración de la integridad acrosomal en espermatozoides de alpaca. Anim Reprod Sci 5: 8792. doi: 10.18548/aspe/ 0002.20

13. Santiani A, Ugarelli A, EvangelistaVargas $S$. 2016. Characterization of functional variables in epididymal alpaca (Vicugna pacos) sperm using imaging flow cytometry. Anim Reprod Sci 173: 49-55. doi: 10.1016/j.anireprosci.2016.08.010

14. Urquieta B, Cepeda R, Cáceres JE, Raggi LA, Rojas JR. 1994. Seasonal variation in some reproductive parameters of male vicuña in the High Andes of northern Chile. J Arid Environ 26: 7987. doi: $10.1006 /$ jare. 1994.1012 
15. Valdivia M, Ruiz M, Bermúdez L, Quinteros S, Gonzales A, Manosalva I, Ponce C, Olazábal J, Dávalos $R$. 1999. Criopreservación de semen de alpacas. En: II congreso Mundial sobre Camélidos. Cusco, Perú.
16. Vojstísková A, Jesina P, Kalous M, Kaplanová V, Houstek J, Tesarová M, Fornusková D, et al. 2004. Mitochondrial membrane potential and ATP production in primary disorders of ATP synthase. Toxicol Mech Methods 14: 711. doi: $10.1080 / 15376520490257347$ 